\title{
Farm Women's Drudgery and Gender Gap Profile: A Participatory Diagnostic Study in District - Sitapur
}

\author{
Saurabh $^{1 *}$, Anand Singh ${ }^{1}$, S.K. Dubey ${ }^{2}$, U.S. Gautam ${ }^{2}$ and Razia Parvez ${ }^{3}$ \\ ${ }^{1}$ Krishi Vigyan Kendra-II, Sitapur, India \\ ${ }^{2}$ ICAR-ATARI (Agricultural Technology Application Research Institute), Zone III, \\ Kanpur, U.P., India \\ ${ }^{3}$ Halina School of Home Science, SHUATS, Allahabad, India \\ *Corresponding author
}

\begin{tabular}{|l|}
\hline Ke y w o r d s \\
$\begin{array}{l}\text { Drudgery, Crop } \\
\text { production, Post harvest } \\
\text { Management, Livestock } \\
\text { management, } \\
\text { Participatory diagnosis, } \\
\text { Gender gap }\end{array}$ \\
\hline Article Info \\
\hline $\begin{array}{l}\text { Accepted: } \\
\text { 22 July } 2018 \\
\text { Available Online: } \\
\text { 10 August } 2018\end{array}$ \\
\hline
\end{tabular}

A B S T R A C T

The role and place of farmwomen in agriculture needed sincere recognition and concerted efforts to address various constraints that they are facing, be it drudgery or other gender related issues. The farm women usually use long static postures while performing farm and allied activities, which increase the static muscular effort resulting in high drudgery, physiological cost, low productivity and low work efficiency. Therefore present study was conducted to find out extent and magnitude of the drudgery and gender gap perceived by farmwomen of the District-Sitapur of central U.P. in three areas namely Crop production, post-harvest and livestock management related works. Total 120 respondents i.e. 40 respondents in each three category were selected through purposive random sampling from across 9 villages spread out in three development blocks. Demographic data and activity profile was recorded using pre-structured interview schedule. The variables like time spent (in minutes), frequency of performance (in man days), degree of difficulty and posture adopted was recorded on 4-point continuum score card. Results showed that harvesting and transplanting was perceived to be most drudgery prone operations in crop production sector. Threshing, cleaning and sieving were intense drudgery loaded activities as perceived in post-harvest management related chores. Cutting fodder from field, chaffing and feeding and watering animal with cleaning of shades were recorded as most strenuous in livestock management category.

\section{Introduction}

The farmwomen perform arduous, tedious and exhaustive works in farm and homestead activities. They are backbone of the agricultural workforce, responsible right from the conservation of seeds to the cooked meal on the plate. Their activities typically include producing agricultural crops, tending animals, processing and preparing food, working for wages in agricultural or other rural enterprises, collecting fuel and water, engaging in trade and marketing, caring for family members and maintaining their homes (Klasen and Lamanna, 2009). World over, about $42 \%$ of women workers are engaged in agriculture while in India, about $60 \%$ of women workers are in agriculture. An interesting feature of 
women's participation in India is the variation across regions (that increases from north to south), socio-cultural and economic groups and across agro-ecological and production systems. Importantly, they carry out a wide range of activities. In India, the work participation rate among women has increased from just $12 \%$ in 1971 to over $25 \%$ in 2001 while among men it has remained just over $51 \%$. High female work participation rate was reflected in more number of women workers in agriculture as evident from increased share of women workers in total agricultural workers that stands at about $40 \%$ (FAO, 2011). Besides some of the agricultural tasks performed by them are not valued adequately and considered less important economically due to multiple roles they perform within the family and the farm. Her access to knowledge and information is constrained and therefore her opportunities get limited (Shivamurthy et al., 2017). Women are lagging behind in the use of improved technology and equipments at farm. This causes significant physical, mental exhaustion and other health problems.

The foremost reason for all these problems are unawareness, hoary techniques of performing task, incompatibility of the technology and attitudinal constraints such as instinctive conservatism and confrontation to change (Singh, et al., 2016). Traditionally women perform exclusively tedious, time and labour intensive works like sowing, transplanting, weeding, intercultural operations, harvesting, threshing, transportation and postharvest operations like shelling, cleaning, grading and processing etc. All these jobs involve considerable amount of drudgery as they are not only done manually but also they have to be performed by bending posture under the shining sun for long hours (Kumar et al., 2011). Women-friendly improved implements and tools need to be developed, refined and evaluated based on feedback of the farm women. This calls for improvement of the present status of rural women in agriculture and to enhance the opportunities for their empowerment. Therefore, present study was conducted to find out the perceived extent of drudgery in the operations of various activities by the farm women in areas of crop production, post-harvest management and livestock management related practices.

\section{Materials and Methods}

The study was carried out in district- Sitapur of central plane of Uttar Pradesh. Two villages Katia and Imlipur were purposively selected as the women are intensely involved in agricultural works in these villages. A sample of 120 respondents was selected who represented as the active workers under agriculture and allied areas from 9 villages spread out in three development blocks. Categorically, 40 respondents again through purposive random sampling were selected each from the group of crop production activities, post-harvest management and livestock management activities to ascertain the intensity of work load among the sample of women farmers. Demographic data, possession of farm tools and implements and data on farm women involved in farm activities was collected by using a prestructured interview schedule. The data were analyzed using the simple statistics of frequency and percentage. The variable workload was recorded as time spent in particular activity in minutes, frequency of performance, total number of performance in man days (total number of hours divided by 8 ), posture used and degree of difficulty on 5 and 4 point continuum and body map.

\section{Results and Discussion}

\section{Socio-economic profile of the farm families}

Data shown in the Table 1 revealed that all the respondents of the study were Hindu (100\%) 
by religion and most of them were belonged to the OBC category $(39.16 \%)$ followed by SC $(31.16 \%)$ and general category $(29.16 \%)$ in their social affiliation. Total 58.33 percent families were nuclear, rest were living in the joint families. Most of families had 5-6 members $(58.33 \%), 19.16$ percent respondents have 7-8 members, while 7.5 percent have above 8 members and 6.66 percent have up to 4 members in their families. There were maximum number of adult males $(27.04 \%)$ and male children $(27.48 \%)$ in the families, while adult women and adult female children were found to be $25.62 \%$ and $19.85 \%$ respectively.

It is evident from the Table 2 that most of the respondents i.e. 111 respondents out of total 120 were found to be small and marginal farmers $(92.5 \%)$ and 7.5 percent were landless farmers. Cent percent of respondents had irrigated farming type with flood type of irrigation method. In case of source of irrigation, only 17.5 percent were owned both bore well and engine, while 66.67 percent respondents families owned engine but water from bore well was borrowed from others, 20.83 percent borrowed both water and engine from others. None of them have other listed irrigation sources. There were four types of crop cycles i.e. Sugarcane + Pulses, paddyWheat-Pulses, Paddy-Wheat-Vegetable and also round the year vegetable production undertaken by the respondent farm families.

Data shown in the Table 3 revealed that the main occupation of the head of the family was agriculture while 72.5 percent also engaged in animal husbandry and agricultural labour (26.7\%). Main source of income was agriculture and allied activities, followed by agriculture wages and allied activities $(28.33 \%)$. Most of the farm families reported to earn more than Rs. 8000 per month of family income whereas 25 percent farm families fall under the range of Rs 5000- 8000/month and only 6.67 earned Rs. 20004000/month.

\section{Possession of farm tools, implements and livestock}

It is evident from the Table 4 that traditional and very basic tools viz. sickle, khurpi, hand hoe, hand plough and manual chaff cutter etc. were possessed by most of them, while very few of them possessed improved tools like maize sheller, chaff cutter, sprinkler, fertilizer broad caster, weeder, hand ridger etc. In case of farm implements also, most of them did not own sophisticated implements. Still, many of possessed diesel engine $(61.67 \%)$, tractor $(29.16 \%)$, sprayers $(19.17 \%)$ and tractor trolley $(17.5 \%)$ are main implements processed by them. Buffalos (33.92\%) and goats $(32.65 \%)$ were most favoured animals, followed by cattle $(21.01 \%)$ and poultry $(8.35 \%)$.

Socio-personal profile, work engagement pattern and work related injuries of women farmers

Details of female respondents engaged in farm families depicted that most of the farm women were from age group 30-40 years (49.16\%) and 34.17 percent were in the age group 40-50 years.92.5\% were illiterate and rest were literate which education qualification, primary $(0.83 \%)$, junior high school (3.33\%), high school $(0.83 \%)$, intermediate $(0.83 \%)$ and graduation and post-graduation degree was possessed by none (Table 4).

Majority (39.17\%) of farm women were conducting household chores, agriculture work in their own farms and dairy works, while $33.33 \%$ each were doing household chores with agricultural work on own land and household chores and dairy work. 28.33 percent of respondent worked as agricultural labour with household chores. 
Table.1 Demographic distribution of farm families of the respondents $(\mathrm{N}=120)$

\begin{tabular}{|c|c|c|c|c|c|}
\hline \multirow[t]{2}{*}{ Parameters } & \multirow{2}{*}{$\begin{array}{c}\text { Crop } \\
\text { Production } \\
n=40\end{array}$} & \multirow{2}{*}{$\begin{array}{c}\text { Dairy and } \\
\text { livestock } \\
\text { production } \\
n=40\end{array}$} & \multirow{2}{*}{$\begin{array}{c}\text { Post-Harvest } \\
\text { Handling } \\
n=40\end{array}$} & \multicolumn{2}{|c|}{ Total $(\mathrm{N}=120)$} \\
\hline & & & & Frequency & $\begin{array}{c}\text { Percent } \\
\text { Distribution }\end{array}$ \\
\hline \multicolumn{6}{|l|}{ Religion } \\
\hline Hindu & $40(100)$ & $40(100)$ & $40(100)$ & 120 & 100 \\
\hline \multicolumn{6}{|l|}{ Caste Category } \\
\hline General & 0 & $17(42.5)$ & $18(45)$ & 35 & 29.16 \\
\hline$\overline{\mathrm{OBC}}$ & $12(30)$ & $21(52.5)$ & $14(35)$ & 47 & 39.16 \\
\hline SC & $28(70)$ & $2(5)$ & $8(20)$ & 38 & 31.16 \\
\hline \multicolumn{6}{|l|}{ Type of Family } \\
\hline Nuclear & $22(55)$ & $23(57.5)$ & $25(62.5)$ & 70 & 58.33 \\
\hline Joint & $18(45)$ & $17(42.5)$ & $15(37.5)$ & 50 & 41.66 \\
\hline \multicolumn{6}{|l|}{ Size of Family } \\
\hline Up to 4 member & - & $5(12.55)$ & $3(7.5)$ & 8 & 6.66 \\
\hline 5 to 6 member & $26(65)$ & $23(57.5)$ & $21(52.5)$ & 70 & 58.33 \\
\hline 7 to 8 member & $10(25)$ & $9(22.5)$ & $4(10)$ & 23 & 19.16 \\
\hline Above 8 member & $4(10)$ & $3(7.5)$ & $2(5)$ & 9 & 7.5 \\
\hline \multicolumn{6}{|c|}{ Family Composition } \\
\hline Adult male & 87 & 82 & 79 & 248 & 27.04 \\
\hline Adult Female & 79 & 86 & 70 & 235 & 25.62 \\
\hline Children Male & 85 & 86 & 81 & 252 & 27.48 \\
\hline Children female & 82 & 51 & 49 & 182 & 19.85 \\
\hline
\end{tabular}

Table.2 Distribution of respondents as per their size of landholding, type of farming, irrigation and major crop cultivated $(\mathrm{N}=120)$

\begin{tabular}{|c|c|c|c|c|c|}
\hline \multirow[t]{2}{*}{ Parameters } & \multirow{2}{*}{$\begin{array}{c}\text { Crop } \\
\text { Production } \\
n=40\end{array}$} & \multirow{2}{*}{$\begin{array}{c}\text { Dairy and } \\
\text { livestock } \\
\text { production } \\
n=40\end{array}$} & \multirow{2}{*}{$\begin{array}{c}\text { Post-Harvest } \\
\text { Handling } \\
\mathbf{n}=40\end{array}$} & \multicolumn{2}{|c|}{ Total $(\mathrm{N}=120)$} \\
\hline & & & & Frequency & $\begin{array}{c}\text { Percent } \\
\text { Distribution }\end{array}$ \\
\hline \multicolumn{6}{|l|}{ Landholding } \\
\hline Landless & $8(20)$ & $1(2.5)$ & 0 & 9 & 7.5 \\
\hline Marginal and small & $32(80)$ & $39(97.5)$ & $40(100)$ & 111 & 92.5 \\
\hline \multicolumn{6}{|l|}{ Type of farming } \\
\hline Irrigated & $40(100)$ & $40(100)$ & $40(100)$ & 120 & 100 \\
\hline \multicolumn{6}{|l|}{ Source of irrigation } \\
\hline $\begin{array}{l}\text { Own Bore Well and } \\
\text { Engine }\end{array}$ & $2(5)$ & $9(22.5)$ & $10(25)$ & 21 & 17.5 \\
\hline $\begin{array}{l}\text { Both Borrowed from } \\
\text { others }\end{array}$ & $12(30)$ & $8(20)$ & $5(12.5)$ & 25 & 20.83 \\
\hline $\begin{array}{l}\text { Owned engine but water } \\
\text { from bore well borrowed }\end{array}$ & $26(65)$ & $23(57.5)$ & $25(62.5)$ & 74 & 61.66 \\
\hline \multicolumn{6}{|l|}{ Irrigation } \\
\hline Flood Irrigation & $40(100)$ & $40(100)$ & $40(100)$ & 120 & 100 \\
\hline Major Crop Cultivated & $\begin{array}{l}\text { 1-Sugarcane +F } \\
\text { 2- Paddy-Whea } \\
\text { 3- Paddy-Whea } \\
\text { 4- Year Round }\end{array}$ & $\begin{array}{l}\text { ses } \\
\text { etable } \\
\text { table Produc }\end{array}$ & & & \\
\hline
\end{tabular}


Table.3 Main and allied occupation, sources and monthly income of the respondents

\begin{tabular}{|c|c|c|c|c|c|}
\hline \multirow[t]{2}{*}{ Parameters } & \multirow{2}{*}{$\begin{array}{c}\text { Crop } \\
\text { Production } \\
n=40\end{array}$} & \multirow{2}{*}{$\begin{array}{c}\text { Dairy and } \\
\text { livestock } \\
\text { production } \\
n=40\end{array}$} & \multirow{2}{*}{$\begin{array}{c}\text { Post-Harvest } \\
\text { Handling } \\
n=40\end{array}$} & \multicolumn{2}{|c|}{ Total $(\mathrm{N}=120)$} \\
\hline & & & & Frequency & $\begin{array}{c}\text { Percent } \\
\text { Distribution }\end{array}$ \\
\hline \multicolumn{6}{|c|}{ Occupation of head of the family } \\
\hline Agriculture & $40(100)$ & $40(100)$ & $40(100)$ & 120 & 100 \\
\hline Ag Labour & $28(70)$ & $4(10)$ & 0 & 32 & 26.7 \\
\hline Business & $1(2.5)$ & $1(2.5)$ & $1(2.5)$ & 3 & 2.5 \\
\hline Service & 0 & $2(5)$ & $1(2.5)$ & 3 & 2.5 \\
\hline Labour & $2(5)$ & 0 & 0 & 2 & 1.67 \\
\hline Animal husbandry & $9(22.5)$ & $40(100)$ & $38(95)$ & 87 & 72.5 \\
\hline \multicolumn{6}{|l|}{ Sources of family Income } \\
\hline Agri+Agri.allied & $9(22.5)$ & $33(82.5)$ & $38(95)$ & 80 & 66.67 \\
\hline Agri.+Business & $1(2.5)$ & $1(2.5)$ & 0 & 2 & 1.6 \\
\hline Agri+ service & 0 & $2(5)$ & $1(2.5)$ & 3 & 2.5 \\
\hline Agri.+Agriallied+Business & 0 & 0 & $1(2.5)$ & 1 & 0.83 \\
\hline Wages+Agri.+Agri allied & $30(75)$ & $4(10)$ & 0 & 34 & 28.33 \\
\hline \multicolumn{6}{|l|}{ Total Monthly Income } \\
\hline $2000-4000$ & $8(20)$ & 0 & 0 & 8 & 6.67 \\
\hline $5000-8000$ & $18(45)$ & $5(12.5)$ & $7(17.5)$ & 30 & 25 \\
\hline$>8000$ & $14(35)$ & $35(87.5)$ & $33(82.5)$ & 82 & 68.33 \\
\hline
\end{tabular}

Table.4 Possession of Farm Tools, Implements and livestock $(\mathrm{N}=120)$

\begin{tabular}{|c|c|c|c|c|c|}
\hline \multirow[t]{2}{*}{ Parameters } & \multirow{2}{*}{$\begin{array}{c}\text { Crop } \\
\text { Production } \\
n=40\end{array}$} & \multirow{2}{*}{$\begin{array}{c}\text { Dairy and } \\
\text { livestock } \\
\text { production } n=40\end{array}$} & \multirow{2}{*}{$\begin{array}{c}\text { Post-Harvest } \\
\text { Handling } \\
n=40\end{array}$} & \multicolumn{2}{|c|}{ Total $(\mathrm{N}=120)$} \\
\hline & & & & Frequency & $\begin{array}{c}\text { Percent } \\
\text { Distribution }\end{array}$ \\
\hline \multicolumn{6}{|l|}{ Farm tools and implements } \\
\hline Sickle & $40(100)$ & $40(100)$ & $40(100)$ & 120 & 100 \\
\hline Khurpi & $40(100)$ & $40(100)$ & $40(100)$ & 120 & 100 \\
\hline Hand Hoe & $40(100)$ & $38(95)$ & $40(100)$ & 118 & 98.33 \\
\hline Hand Plough(phawda) & $40(100)$ & $40(100)$ & $40(100)$ & 120 & 100 \\
\hline Maize sheller & $6(15)$ & $2(5)$ & $2(5)$ & 10 & 8.33 \\
\hline Chaff Cutter & $4(10)$ & $25(62.5)$ & $28(70)$ & 57 & 47.5 \\
\hline Sprinkler & - & $2(5)$ & $6(15)$ & 8 & 6.67 \\
\hline Manual Chaf cutter (Gandasa) & $5(12.5)$ & $13(32.5)$ & $12(30)$ & 30 & 25.0 \\
\hline Fertilizer sprayer/Broad Caster & $5(12.5)$ & $12(30)$ & $24(60)$ & 41 & 34.17 \\
\hline \multicolumn{6}{|l|}{ Farm Implements } \\
\hline Tractor & $2(5)$ & $18(45)$ & $15(37.5)$ & 35 & 29.16 \\
\hline Trolly & $1(2.5)$ & $9(22.5)$ & $11(27.5)$ & 21 & 17.5 \\
\hline Engine & $26(65)$ & $23(57.5)$ & $25(62.5)$ & 74 & 61.67 \\
\hline Cultivator & - & $1(2.5)$ & $1(2.5)$ & 2 & 1.6 \\
\hline Harrow & $2(5)$ & $1(2.5)$ & $5(12.5)$ & 8 & 6.67 \\
\hline Winnowing fan & 0 & $1(2.5)$ & $1(2.5)$ & 2 & 1.6 \\
\hline Others (Sprayer) & $6(15)$ & $12(30)$ & $5(12.5)$ & 23 & 19.17 \\
\hline \multicolumn{6}{|l|}{ Livestock possession (No.) } \\
\hline Cow & 21 & 33 & 29 & 83 & 21.01 \\
\hline Buffalo & 9 & 119 & 6 & 134 & 33.92 \\
\hline Goat & 95 & 9 & 25 & 129 & 32.65 \\
\hline Poultry & 33 & 0 & 0 & 33 & 8.35 \\
\hline Pigs & 11 & 0 & 0 & 11 & 2.78 \\
\hline Pigeon & 5 & 0 & 0 & 5 & 1.26 \\
\hline
\end{tabular}


Table.5 Details of female respondents engaged in farm activities $(\mathrm{N}=120)$

\begin{tabular}{|c|c|c|c|c|c|c|}
\hline \multirow[t]{2}{*}{ SI. No. } & \multirow[t]{2}{*}{ Parameters } & \multirow{2}{*}{$\begin{array}{c}\text { Crop } \\
\text { Production } \\
n=40\end{array}$} & \multirow{2}{*}{$\begin{array}{c}\text { Dairy and } \\
\text { livestock } \\
\text { production } n=40\end{array}$} & \multirow{2}{*}{$\begin{array}{c}\text { Post- } \\
\text { Harvest } \\
\text { Handling } \\
n=40\end{array}$} & \multicolumn{2}{|c|}{ Total $(\mathrm{N}=120)$} \\
\hline & & & & & Frequency & $\begin{array}{c}\text { Percent } \\
\text { Distribution }\end{array}$ \\
\hline \multirow[t]{5}{*}{1} & \multicolumn{4}{|l|}{ Age of the respondents } & & \\
\hline & $20-30$ & $2(5)$ & $6(15)$ & $5(12.5)$ & 13 & 10.83 \\
\hline & $30-40$ & $20(50)$ & $14(35)$ & $25(62.5)$ & 59 & 49.16 \\
\hline & $40-50$ & $15(37.5)$ & $18(45)$ & $8(20)$ & 41 & 34.17 \\
\hline & above 50 & $3(7.5)$ & $2(5)$ & $2(5)$ & 7 & 5.83 \\
\hline \multirow[t]{6}{*}{2} & \multicolumn{4}{|l|}{ Education } & & \\
\hline & Illiterate & $39(97.5)$ & $37(92.5)$ & $35(87.5)$ & 111 & 92.5 \\
\hline & Primary & $1(2.5)$ & 0 & 0 & 1 & 0.83 \\
\hline & Junior High School & 0 & $2(5)$ & $2(5)$ & 4 & 3.33 \\
\hline & High School & 0 & 0 & $1(2.5)$ & 1 & 0.83 \\
\hline & Intermediate & 0 & $1(2.5)$ & & 1 & 0.83 \\
\hline \multirow[t]{6}{*}{3} & \multicolumn{4}{|l|}{ Occupation } & & \\
\hline & $\begin{array}{l}\text { Housewife and Agri } \\
\text { work (AW) }\end{array}$ & $40(100)$ & 0 & 0 & 40 & 33.33 \\
\hline & $\begin{array}{l}\mathrm{HW}+\mathrm{Ag} \text { Labour/ } \\
\text { Labour }\end{array}$ & $30(75)$ & $4(10)$ & 0 & 34 & 28.33 \\
\hline & $\mathrm{HW}+\mathrm{AW}+\mathrm{AH}$ & $9(22.5)$ & 0 & $38(95)$ & 47 & 39.17 \\
\hline & $\begin{array}{l}\text { HW+ AW+ Bussiness/ } \\
\text { Service }\end{array}$ & $1(2.5)$ & $3(7.5)$ & $2(5)$ & 6 & 5.00 \\
\hline & HW+Dairy Farming & 0 & $40(100)$ & 0 & 40 & 33.33 \\
\hline \multirow[t]{4}{*}{4} & \multicolumn{4}{|l|}{ The duration of work } & & \\
\hline & 8 am To 6 pm & $8(20)$ & $5(12.5)$ & $7(17.5)$ & 20 & 16.67 \\
\hline & $\begin{array}{l}\text { Morning and Evening } \\
\text { Pattern }\end{array}$ & $32(80)$ & $18(45)$ & $33(82.5)$ & 83 & 69.17 \\
\hline & $\begin{array}{l}\text { Early Morning till Late } \\
\text { Night }\end{array}$ & 0 & $12(30)$ & 0 & 12 & 10.00 \\
\hline \multirow[t]{5}{*}{5} & \multicolumn{4}{|c|}{ Mode of payment of Wage } & & \\
\hline & Daily-cash/In kind & $5(12.5)$ & 0 & 0 & 5 & 14.70 \\
\hline & Weekly-cash/In kind & $23(57.5)$ & $2(5)$ & 0 & 25 & 73.53 \\
\hline & Fortnightly/Cash & $2(5)$ & $2(5)$ & 0 & 4 & 11.76 \\
\hline & Monthly-cash/In kind & 0 & 0 & 0 & 0 & 0 \\
\hline \multirow[t]{4}{*}{11} & \multicolumn{4}{|c|}{ Tools helps in reducing the force } & & \\
\hline & Yes & $12(30)$ & $2(5)$ & $5(12.5)$ & 19 & 15.83 \\
\hline & No & $23(57.5)$ & $24(60)$ & $9(22.5)$ & 56 & 46.67 \\
\hline & Uncertain & $5(12.5)$ & $14(35)$ & $26(65)$ & 45 & 37.50 \\
\hline \multirow[t]{3}{*}{12} & \multicolumn{4}{|c|}{ Feel Tired after performing work } & & \\
\hline & Yes & $40(100)$ & $40(100)$ & $40(100)$ & 120 & 100 \\
\hline & No & & & & & \\
\hline \multirow[t]{3}{*}{13} & Facing joint problems i & farm/ allied acti & & & & \\
\hline & Yes & $40(100)$ & $40(100)$ & $40(100)$ & 120 & 100 \\
\hline & No & & & & & \\
\hline 14 & Feel difficulty in bendir & in farm/ allied a & & & & \\
\hline & Yes & $40(100)$ & $40(100)$ & $40(100)$ & 120 & 100 \\
\hline & No & - & & & & \\
\hline 15 & Got Scratches/ cuts/ inj & ries in farm/ allic & vities & & & \\
\hline & Yes & $40(100)$ & $40(100)$ & $40(100)$ & 120 & 100 \\
\hline & No & & & & & \\
\hline 16 & Suffered from diseases/ & ealth problems & farm/ allied activi & & & \\
\hline & Eye infections & $21(52.5)$ & $2(5)$ & $29(72.5)$ & 52 & 43.33 \\
\hline & $\begin{array}{l}\text { Ear disorders/ hearing } \\
\text { problems }\end{array}$ & $5(12.5)$ & - & $2(5)$ & 7 & 5.83 \\
\hline & $\begin{array}{l}\text { Skin cuts/ Fungal } \\
\text { infection }\end{array}$ & $40(100)$ & $19(47.5)$ & $27(67.5)$ & 86 & 71.67 \\
\hline & Allergy & $40(100)$ & $15(37.5)$ & $40(100)$ & 95 & 79.17 \\
\hline
\end{tabular}




\begin{tabular}{|c|c|c|c|c|c|c|}
\hline & $\begin{array}{l}\text { Heat exhaustion/ Heat } \\
\text { strokes }\end{array}$ & $40(100)$ & $40(100)$ & $40(100)$ & 120 & 100 \\
\hline & $\begin{array}{l}\text { Breathing problems/ } \\
\text { Bronchitis/ Asthma }\end{array}$ & $24(60)$ & $38(95)$ & $40(100)$ & 102 & 85 \\
\hline & $\begin{array}{l}\text { Numbness in } \\
\text { feets/hands }\end{array}$ & $40(100)$ & $40(100)$ & $40(100)$ & 120 & 100 \\
\hline & Back ache & $40(100)$ & $40(100)$ & $40(100)$ & 120 & 100 \\
\hline & Head ache & $40(100)$ & $40(100)$ & $40(100)$ & 120 & 100 \\
\hline & $\begin{array}{l}\text { Pain in Knees, } \\
\text { shoulders and other } \\
\text { joints }\end{array}$ & $40(100)$ & $40(100)$ & $40(100)$ & 120 & 100 \\
\hline & Blisters on palm & $40(100)$ & $12(30)$ & $40(100)$ & 92 & 76.67 \\
\hline & Any other & & & & & \\
\hline 17 & \multicolumn{6}{|c|}{ To what extent you adopted one posture in farm/ allied activities } \\
\hline & $30 \mathrm{~min}$ & $7(17.5)$ & $8(20)$ & $5(12.5)$ & 20 & 16.67 \\
\hline & $1 \mathrm{hr}$ & $13(32.5)$ & $25(62.5)$ & $16(40)$ & 54 & 45.00 \\
\hline & $2 \mathrm{hr}$ & $11(27.5)$ & $6(15)$ & $13(32.5)$ & 30 & 25.00 \\
\hline & Above $2 \mathrm{hr}$ & $9(22.5)$ & $1(2.5)$ & $6(15)$ & 16 & 13.33 \\
\hline \multirow[t]{5}{*}{18} & \multicolumn{6}{|c|}{ Do you go to the doctor after injuries/ allergies/ infections } \\
\hline & \multicolumn{6}{|l|}{ Always } \\
\hline & \multicolumn{6}{|l|}{ Sometimes } \\
\hline & $\begin{array}{l}\text { Only when condition is } \\
\text { serious }\end{array}$ & $12(30)$ & $29(72.5)$ & $31(77.5)$ & 72 & 60.00 \\
\hline & Never & $28(70)$ & $11(27.5)$ & $9(22.5)$ & 48 & 40.00 \\
\hline 19 & \multicolumn{6}{|c|}{ How frequently you take care of hand tools and implements } \\
\hline & Yes & $40(100)$ & $40(100)$ & $40(100)$ & 120 & 100 \\
\hline & No & 0 & 0 & 0 & 0 & 0 \\
\hline & Once in a month & 0 & 0 & 0 & 0 & 0 \\
\hline & Before use & 0 & 0 & 0 & 0 & 0 \\
\hline & When needed & $40(100)$ & $40(100)$ & $40(100)$ & 120 & 100 \\
\hline
\end{tabular}

Table.6 Major area of work participation of women and men farmers

\begin{tabular}{|c|c|c|}
\hline CATEGORY & $\begin{array}{l}\text { OPERATIONS/ACTIVITIES PERFORMED BY } \\
\text { WOMEN }\end{array}$ & $\begin{array}{l}\text { OPERATIONS PERFORMED BY } \\
\text { MEN }\end{array}$ \\
\hline $\begin{array}{l}\text { Crop } \\
\text { Production }\end{array}$ & $\begin{array}{ll}\text { - } & \text { Cleaning of field: Removal of Stalks and stubbles } \\
\text { - } & \text { Nursery bed preparation } \\
\text { - } & \text { Seed Sowing/ Dibbling } \\
\text { - } & \text { Paddy Transplanting } \\
\text { - } & \text { Sugarcane Seed Cutting and planting } \\
\text { - } & \text { Gap filling } \\
\text { - } & \text { Field Cleaning and Weeding } \\
\text { - } & \text { Harvesting/ Plucking/ Uprooting/ Detopping etc. } \\
\text { - } & \text { Bundling and carrying to threshing place }\end{array}$ & $\begin{array}{l}\text { - } \quad \text { Ploughing and making of fields } \\
\text { - } \quad \text { Seed purchase and Nursery sowing } \\
\text { - } \text { Irrigation, fertilizer purchase and } \\
\text { application } \\
\text { - } \quad \text { Helps in harvesting etc. }\end{array}$ \\
\hline $\begin{array}{l}\text { Post-Harvest } \\
\text { Handling }\end{array}$ & $\begin{array}{ll}\text { - } & \text { Crushing } \\
\text { - } & \text { Threshing/ Decortication/ Shelling } \\
\text { - } & \text { Winnowing and Sun Drying } \\
\text { - } & \text { Baging, Grading and Cleaning } \\
& \text { Band stitching of bags for Storage }\end{array}$ & $\begin{array}{l}\text { - } \text { Arrangements for thresher } \\
\text { - Helps in winnowing } \\
\text { - } \quad \text { Seading of bags for transportation } \\
\text { - }\end{array}$ \\
\hline $\begin{array}{l}\text { Dairy and } \\
\text { Livestock } \\
\text { Production }\end{array}$ & $\begin{array}{l}\text { - } \quad \text { Milking of animals } \\
\text { - } \quad \text { Fodder cutting/ carrying } \\
\text { - } \quad \text { Cleaning of animal shed } \\
\text { - } \quad \text { Carrying of animal waste for disposal }\end{array}$ & $\begin{array}{ll}\text { - } & \text { Sell and purchase of animals } \\
\text { - } & \text { Fodder cultivation } \\
\text { - } & \text { Vaccination and medication } \\
\text { - Selling of milk }\end{array}$ \\
\hline
\end{tabular}


Table.7 Average time spent (in hours/person/day season wise) by the farm women in the major activities and major activities in which women perceived drudgery

\begin{tabular}{|c|c|c|c|c|c|c|}
\hline $\begin{array}{l}\text { SI. } \\
\text { No. }\end{array}$ & Activity & Season & $\begin{array}{l}\text { Time spend (hrs/ } \\
\text { person/ day) }\end{array}$ & $\begin{array}{l}\text { Frequency of } \\
\text { performance }\end{array}$ & Posture used & $\begin{array}{l}\text { Drudgery level } \\
\text { Perceived }\end{array}$ \\
\hline \multicolumn{7}{|c|}{ Crop Production related work areas: } \\
\hline & \multirow{3}{*}{$\begin{array}{l}\text { Cleaning of field: Removal of } \\
\text { Stalks and stubbles }\end{array}$} & Rabi & \multirow[t]{3}{*}{$3-4 \mathrm{hrs}$} & \multirow[t]{3}{*}{ According to crop } & \multirow[t]{3}{*}{ Sitting + Bending } & \multirow[t]{3}{*}{ Difficult } \\
\hline & & Kharif & & & & \\
\hline & & Zaid & & & & \\
\hline & \multirow[t]{3}{*}{ Nursery bed preparation } & Rabi & \multirow[t]{3}{*}{$3-4 \mathrm{hrs}$} & \multirow{3}{*}{$\begin{array}{l}\text { According to } \\
\text { vegetable }\end{array}$} & \multirow[t]{3}{*}{ Sitting + Bending } & \multirow[t]{3}{*}{ Difficult } \\
\hline & & Kharif & & & & \\
\hline & & Zaid & & & & \\
\hline & \multirow[t]{3}{*}{ Seed Sowing/ Dibbling } & Rabi & \multirow[t]{3}{*}{$3-4 \mathrm{hrs}$} & \multirow[t]{3}{*}{ According to crop } & \multirow[t]{3}{*}{ Standing + Bending } & \multirow[t]{3}{*}{ Difficult } \\
\hline & & Kharif & & & & \\
\hline & & Zaid & & & & \\
\hline & \multirow[t]{3}{*}{ Vegetable Sapling Transplanting } & Rabi & \multirow[t]{3}{*}{$3-4 \mathrm{hrs}$} & \multirow{3}{*}{$\begin{array}{l}\text { According to } \\
\text { vegetable }\end{array}$} & \multirow[t]{3}{*}{ Sitting + Bending } & \multirow[t]{3}{*}{ Difficult } \\
\hline & & Kharif & & & & \\
\hline & & Zaid & & & & \\
\hline & Paddy Transplanting & Kharif & $6 \mathrm{hrs}$. & Yearly & Standing + Bending & Very Difficult \\
\hline & \multirow{2}{*}{$\begin{array}{l}\text { Sugarcane Seed Cutting and } \\
\text { planting }\end{array}$} & Autumn & \multirow[t]{2}{*}{$6 \mathrm{hrs}}$. & \multirow[t]{2}{*}{ Twice in year } & \multirow{2}{*}{$\begin{array}{l}\text { Bending }+ \\
\text { Sitting(cutting) and } \\
+ \text { Standing } \\
\text { (planting) }\end{array}$} & Difficult \\
\hline & & Spring & & & & \\
\hline & Gap filling & Rabi & $3-4 \mathrm{hrs}$ & According to crop & Sitting + Bending & Neither \\
\hline & & Kharif & & & & Difficult nor \\
\hline & & Zaid & & & & \\
\hline & Field Cleaning and Weeding & Rabi & $6 \mathrm{hrs}$. & 1-2 Times/ Season & Sitting + Bending & Difficult \\
\hline & & Kharif & & & & \\
\hline & & Zaid & & & & \\
\hline & Harvesting/ Plucking/ Uprooting/ & Rabi & For Crops: & Multiple times & Sitting + Bending & Very Difficult \\
\hline & Detopping etc & Kharif & 5-6 hrs. & according to crop & & \\
\hline & & Zaid & $\begin{array}{l}\text { For Veg: } \\
\qquad 2-4 \text { hrs }\end{array}$ & and vegetable & & \\
\hline & Bundling and carrying to & Rabi & $1-2 \mathrm{hrs}$ & Multiple times & Sitting + Bending & Neither \\
\hline & threshing place & Kharif & & according to crop & & Difficult nor \\
\hline & & Zaid & & and vegetable & & Easy \\
\hline 2. & Post-Harvest Handling related & vork areas: & & & & \\
\hline & Crushing & All season & $2-3 \mathrm{hrs}$ & According to crop & Sitting + Bending & $\begin{array}{l}\text { Neither } \\
\text { Difficult nor } \\
\text { Easy }\end{array}$ \\
\hline & $\begin{array}{l}\text { Threshing/ Decortication/ } \\
\text { Shelling }\end{array}$ & All season & $4-5 \mathrm{hrs}$ & According to crop & Sitting + Bending & Difficult \\
\hline & Winnowing and Sun Drying & All season & $2-3 \mathrm{hrs}$ & According to crop & $\begin{array}{l}\text { Standing }+ \text { Sitting }+ \\
\text { Bending }\end{array}$ & Difficult \\
\hline & Sieving, Grading and Cleaning & All season & $2-3 \mathrm{hrs}$ & According to crop & Sitting + Bending & Difficult \\
\hline & $\begin{array}{l}\text { Bagging and stitching of bags for } \\
\text { Storage }\end{array}$ & All season & $1-3 \mathrm{hrs}$ & According to crop & $\begin{array}{l}\text { Standing }+ \text { Sitting }+ \\
\text { Bending }\end{array}$ & $\begin{array}{l}\text { Neither } \\
\text { Difficult nor } \\
\text { Easy }\end{array}$ \\
\hline 3. & Dairy and Livestock Productio & related work & eas: & & & \\
\hline & Milking of animals & All season & $0.5-1 \mathrm{hr}$ & Daily & Sitting + Bending & $\begin{array}{l}\text { Neither } \\
\text { Difficult nor } \\
\text { Easy }\end{array}$ \\
\hline & Fodder cutting/ carrying & All season & $1-2 \mathrm{hrs}$ & Daily & $\begin{array}{l}\text { Standing }+ \text { Sitting }+ \\
\text { Bending }\end{array}$ & Difficult \\
\hline & $\begin{array}{l}\text { Fodder preparation and feeding to } \\
\text { animals }\end{array}$ & All season & $1-2 \mathrm{hrs}$ & Daily & Standing + Bending & $\begin{array}{l}\text { Neither } \\
\text { Difficult nor } \\
\text { Easy }\end{array}$ \\
\hline & Cleaning of animal shed & All season & $1-2 \mathrm{hrs}$ & Daily & Sitting + Bending & Difficult \\
\hline & $\begin{array}{l}\text { Carrying of animal waste for } \\
\text { disposal }\end{array}$ & All season & $1 \mathrm{hr}$ & Daily & Standing + Bending & Difficult \\
\hline
\end{tabular}


Farm women are engaged in each and every activity of household as well as on farms thus the working pattern of respondents was found to be morning till night pattern (69.17\%). About $16.67 \%$ followed the work timing of $8 \mathrm{am}$ to $6 \mathrm{pm}$ pattern because they were agricultural labours. In case of comfortability of farming tools in use, $70.83 \%$ found them comfortable, while $51.67 \%$ of respondents were uncertain if there is any need of change in tools. Regarding heaviness of tools, majority $(69.17 \%)$ of respondents felt that the tools they were using were comfortable. They do not require any change in tool (19.17\%). The reason behind the response was may be, as they were not in the acquaintance of new tools. Respondents were not aware about the availability of any kind of drudgery reducing tools too. Cent percent farm women faced one or the other injury during various farm operation and $100 \%$ said that their sickle's handle was made up of wooden which also caused injuries when worked with them for long period.

All of the farmwomen felt tired while performing different farm activities. All the respondents $(100 \%)$ faced joint problems in farm operations, felt difficulty in bending in farms and all respondents got some or the kind of scratches on body during agricultural works. Most of the respondents suffered from heat exhaustion and heat strokes, numbness in hand/feet, backache, headache, pain in knees, shoulders, joints $(100 \%)$, breathing problems $(85 \%)$, skin diseases like allergies $(79.17 \%)$, blisters on palms $(76.67 \%)$ and fungal infections (71.67\%). They worked in bending posture for hours continuously under scorching sun in urea laced water while transplanting of paddy which seriously injured their feet skin and caused various ailments. As majority of respondents were agricultural labor worked under contractor so they have to adopt one posture for more than 30 minutes $(83.33 \%)$. It was observed they stand for one or two minutes but again adopt the same posture. Care and maintenance of farm implements was taken by males usually whenever needed (Table 4).

\section{Activity profile of farm women and perceived drudgery}

While observing the activity profile of the farm families involved in crop production and related works it was found that major areas of work participation of women farmers were cleaning of field, removal of Stalks and stubbles, nursery bed preparation, seed Sowing/ dibbling, vegetable sapling transplanting, paddy transplanting, sugarcane seed cutting and planting, gap filling, field cleaning and weeding, harvesting/ plucking/ uprooting/ detopping etc., bundling and carrying to threshing place. In case of postharvest management activities women were mainly involved in crushing, threshing/ decortication/ shelling, winnowing and sun drying, sieving, grading and cleaning, bagging and stitching of bags for storage.

It is also evident from the Table 5 that women are involved in most of the work related to the Livestock management like milking of animals, fodder cutting/ carrying, fodder preparation and feeding to animals, cleaning of animal shed, carrying of animal waste for disposal. It is obvious from the Table 5 that though men are involved in various heavy operations in crop production, post-harvest and dairy management categories, but they also have access to improved machineries and tools to perform those operations. However farm women are engaged in every tedious, laborious and monotonous activity in each category that also with the help of very traditional basic types of the farm tools, even than they do not have decision making power and access to the money. This leads to the gender discrepancy, high drudgery and low efficiency on the part of farmwomen. 
Average time spent (in hours/person/day season wise) by the farm women in the major activities and major activities in which women perceived drudgery

It is evident from the Table 6 that in crop production category the respondents perceived very difficult level of drudgery on the scale in paddy transplanting which is done in kharif season mostly adopting bending posture with standing for 1-3 minutes. Same is the case for Harvesting/ Plucking/ Uprooting/ Detopping etc. which is to be done multiple times in a year according to the crops grown. Mostly sitting and bending posture was adopted by respondents while performing various activities in each category. Whereas other activities were fall under difficult and neither difficult nor easy drudgery level of the scale (Table 7).

It can be concluded from the results of the study that crop production, post-harvest handling and dairy and livestock production related work were drudgery prone usually done by using traditional types of the tools causes various health problems. Farmwomen were unaware of women specific improved farm tools/ implements and their availability in the area is also a major issue. There is gender gap in work profile and decision making process of the farm families. Work rest cycle, various yoga techniques, women specific tools and implements can be suggested to reduce the effect of drudgery on the farm women while improving their work efficiency. Knowledge and skill based trainings of farm women in related areas must be given for their capacity building.

\section{References}

Klasen, S., and Lamanna, F. (2009). The impact of gender inequality in education and employment on economic growth: new evidence for a panel of countries. Feminist Economics, 15(3): 91-132.

Kumar Bharath, T.P., Gowda, G. V. and Khandekar, N. (2011). Time utilization pattern and drudgery of horticultural crops. International Journal of Engineering and Management Sciences 2(2): 93-96.

Shivamurthy, M., Rani S. and Prabhuswamy, Y.H. (2017). Perception of farm women about efficiency of drudgery-reducing farm implements. International Journal of Farm Sciences 7(1): 29-32

Singh, S., Ahlawat, S., Sanwal S., Ahlawat, T.R. and Gora A. (2016).Drudgery reduction of farm women through improved tools. International Journal of Agriculture Sciences. 8(14): 1242-1249

The State of Food and Agriculture: Women in Agriculture- Closing the gender gap for development (2011). Food and Agriculture Organization of the United Nations. Rome. Pp-146

\section{How to cite this article:}

Saurabh, Anand Singh, S.K. Dubey, U.S. Gautam and Razia Parvez. 2018. Farm Women's Drudgery and Gender Gap Profile: A Participatory Diagnostic Study in District - Sitapur. Int.J.Curr.Microbiol.App.Sci. 7(08): 3984-3993. doi: https://doi.org/10.20546/ijcmas.2018.708.412 